\title{
Vocal tract adjustments in the high soprano range
}

\author{
Maëva Garnier \\ School of Physics, University of New South Wales, Sydney, New South Wales 2052, Australia \\ Nathalie Henrich \\ Department of Speech and Cognition, GIPSA-Lab, 38402 Saint Martin d'Héres, France (UMR 5216: CNRS, \\ Grenoble Universities) \\ John Smith and Joe Wolfe \\ School of Physics, University of New South Wales, Sydney, New South Wales 2052, Australia
}

(Received 1 November 2009; revised 8 April 2010; accepted 8 April 2010)

Twelve sopranos with different levels of expertise (4 nonexperts, 4 advanced, 4 professionals) sustained pitches from A4 $(\sim 440 \mathrm{~Hz})$ to their highest pitch (ranging from C6 to D7, i.e., from $\sim 1000$ to $2300 \mathrm{~Hz})$. The frequencies of their first two vocal tract resonances $(R 1$ and $R 2)$ were measured by broadband excitation at the mouth and compared with the voice harmonics $\left(f_{0}, 2 f_{0}\right.$, etc). Lip articulation was measured from simultaneous video recordings. Adjustment of $R 1$ near to $f_{0}\left(R 1: f_{0}\right.$ tuning $)$ was observed below C6 to D6 $(\sim 1000-1200 \mathrm{~Hz})$ for both expert and non-expert singers. Experts began this tuning at lower pitches. Some singers combine $R 2: 2 f_{0}$ adjustment with $R 1: f_{0}$ tuning. Some singers increased mouth area with increasing pitch over the whole $R 1: f_{0}$ tuning range. Other singers showed this strategy on the higher part of the $R 1: f_{0}$ range only, and used another, as yet unidentified, articulatory strategy on the lower part. To achieve very high pitches, some singers extended the range of $R 1: f_{0}$ tuning as far as E6 to F\#6 $(\sim 1300-1500 \mathrm{~Hz})$ while others adjusted $R 2$ near $f_{0}$ over the highest pitch range.

(C) 2010 Acoustical Society of America. [DOI: 10.1121/1.3419907]

PACS number(s): 43.75.Rs [DAB]

Pages: $3771-3780$

\section{INTRODUCTION}

The vocal range of sopranos can extend over two to three octaves. Different voice registers within this range can be perceived by the listener ${ }^{1,2}$ and are related to differences in use of the larynx and the vocal tract. ${ }^{3,4}$ High registers of the soprano voice are not very well understood yet. This study investigates vocal tract adjustments (acoustic resonances, lip articulation) in the high and very high ranges of the soprano voice with the aim (1) to better understand their interest in terms of acoustic coupling between the tract and the glottal source, (2) to identify pitch ranges with different behaviors and (3) to examine the influence of expertise.

The most salient and studied register transition in the soprano voice, known as the primo passagio by singing voice teachers, occurs in the low to medium pitch range of the female voice, between G3 $(\sim 200 \mathrm{~Hz})$ and G4 $(\sim 400 \mathrm{~Hz}) .{ }^{3,5}$ It corresponds to a laryngeal transition ${ }^{6}$ between the two main laryngeal mechanisms: $:^{3,7}$ M1 (commonly used to produce the chest' register) and M2 (commonly used to produce the female 'head' register). M1 and M2 mainly differ by the vibrating mass in action: in M1, the deeper layer (vocalis muscle) participates in the vibrating mass in action, which is not the case in M2. Classically trained sopranos learn to lower the limit of their M2 range so they can cover their standard range in M2 only, thus avoiding the primo passagio at low pitch.

The register transitions at higher pitches have been less studied. There appears to be no general consensus about the registers of the female singing voice above the primo passagio. The number, the range and the characteristics of these registers appear to vary with singing techniques, voice classification and voice training. There is some agreement that sopranos modify articulation around C5 to E5 ( 500 to 650 $\mathrm{Hz})^{8-10}$ and some researchers associate this with a transition from a "middle" register to an "upper" register. ${ }^{4}$ Also, many authors agree to distinguish a "whistle" or "flageolet" register in the highest part of the female voice, above C6 or E6 $(>1000$ to $1300 \mathrm{~Hz}) .{ }^{11}$ Many untrained or non-expert singers have difficulty controlling their high range: some cannot sing it at all, others exhibit decreased efficiency, breathy voice quality and/or voice instabilities.

One technique observed in classically trained sopranos is to increase the mouth opening with increasing pitch over a range whose lower limit typically lies between B4 $(\sim 500 \mathrm{~Hz})$ and E5 $(\sim 650 \mathrm{~Hz})$, depending on the vowel, and whose upper limit is often around C6 $(\sim 1050 \mathrm{~Hz}){ }^{8-10}$ Within this range, direct measurements of the resonances of the vocal tract have shown that classically trained sopranos adjust the frequency $R 1$ of the first resonance so that it lies close to the first voice harmonic $\left(f_{0}\right) .{ }^{12-14}$ This is referred to as $R 1: f_{0}$ tuning. As $R 1$ is positively correlated with jaw lowering, ${ }^{15}$ and as this articulatory movement increases the open area of the mouth, the increase of mouth opening observed in the high soprano range is believed to be an important articulatory strategy to achieve this $R 1: f_{0}$ tuning. However, the relationship between changes in mouth area and $R 1$ has not, to our knowledge, been experimentally quantified.

The $R 1: f_{0}$ tuning is thought to improve voice efficiency: when a voice harmonic lies in the proximity of a vocal tract resonance, its radiation is enhanced, which is reported to 


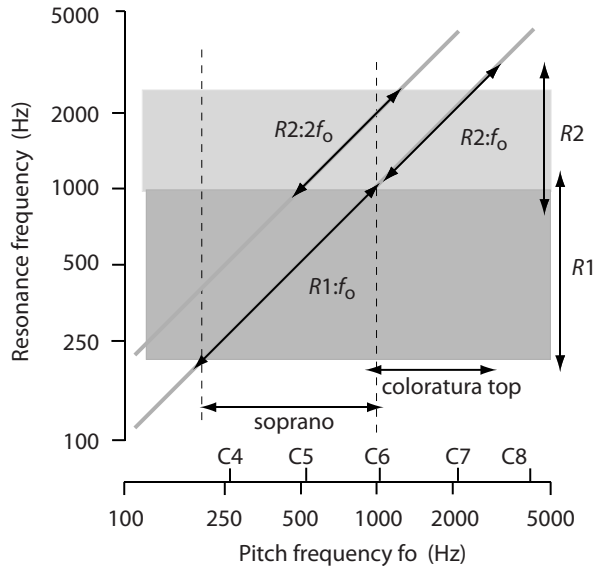

FIG. 1. A schematic showing the typical ranges of the vocal tract resonances $R 1$ and $R 2$. The diagonal, double-headed arrows on the graph show regions in which different tuning strategies $\left(R 1: f_{0}, R 2: f_{0}, R 2: 2 f_{0}\right)$ might be practiced.

increase sound pressure level, up to $20 \mathrm{~dB} \cdot{ }^{16,17}$ Further, according to one theoretical model, transfer of energy from the glottal source to the tract is optimized if the input impedance of the tract is inertive at $f_{0}$, i.e., if the first harmonic lies slightly below the frequency of a vocal tract resonance. ${ }^{18,19}$ Supporting this model is the weakening in vocal fold vibration observed in female singers when $f_{0}$ exceeds $R 1{ }^{11,20} \mathrm{Be}-$ cause $R 1: f_{0}$ tuning has been studied in trained singers producing high sound levels, it is sometimes considered an expert technique learnt by some sopranos during classical vocal training. However, a mouth opening that increases with rising pitch has also been reported in a non-classically trained soprano ${ }^{21}$ and little difference was observed in mouth opening between expert and non-expert sopranos. ${ }^{22}$ For this reason, it is interesting to compare the extent of $R 1: f_{0}$ tuning among singers with different degrees of vocal training.

Although resonance tuning has been demonstrated below $1050 \mathrm{~Hz}(\mathrm{C} 6)$, the situation at higher frequencies has not yet been investigated. Considerable variability in timbre is reported among the minority of singers who sing this range: Sounds may be "breathy," "tight" or "tiny," with reduced intensity and dynamics, or they may be "loud" and "bright," "with optimal vocal folds adduction." ${ }^{23}$ In particular, coloratura sopranos are relatively comfortable above C6 to D6 $(\sim 1100 \mathrm{~Hz})$. Could it be that coloratura singers have learnt specific resonance strategies to sing the top of their range?

Figure 1 presents a simplified schematic that plots resonance frequency against $f_{0}$ and shows the approximate regions where vocal tract resonances $R 1$ and $R 2$ could be matched to $f_{0}$. Three possible strategies for resonance tuning are immediate candidates.

A first possibility is that the $R 1: f_{0}$ tuning could be extended to frequencies above $1050 \mathrm{~Hz}$, thereby maintaining $R 1$ near or above $f_{0}$. However, this raises the problem that $R 1$ would then exceed the reported values for normal female speech (see Fig. 1). Furthermore, the mouth opening for sopranos singing their high $\mathrm{C}(\mathrm{C} 6 \sim 1050 \mathrm{~Hz})$ is already large, so it is not clear how $R 1: f_{0}$ tuning could be extended. Could the mouth be opened in different ways? Could other articulatory changes be involved? Might such singers just have shorter or at least different vocal tracts?

A second strategy that would maintain resonance tuning above about C6 $\left(f_{0}>1050 \mathrm{~Hz}\right)$ involves matching $R 2$ rather than $R 1$ with $f_{0}$, particularly as $R 2$ can typically range from 1000 to $2500 \mathrm{~Hz}$ (see Fig. 1). Perhaps expert singers maintain resonance tuning above C6 to D6 by adjusting $R 2$ to $f_{0}$ once the first harmonic has passed the possible range of $R 1$.

A third strategy would involve matching $R 2$ with $2 f_{0}$ rather than $f_{0}$. The normal upper limit of $R 2$ around $2500 \mathrm{~Hz}$ would limit this $R 2: 2 f_{0}$ tuning to pitches below E6 $(\sim 1300 \mathrm{~Hz})$, but it might act as a useful technique in the intermediate region between $R 1: f_{0}$ and $R 2: f_{0}$ tuning - see Fig. 1 . It is also possible that $R 2: 2 f_{0}$ tuning could be maintained in parallel with $R 1: f_{0}$ tuning, with possible benefits in increased sound level and stability.

In this study, the various strategies for singing at very high pitch were investigated by measuring the first two vocal tract resonances, along with lip articulation, when 12 sopranos with different levels of expertise sang from A4 $(440 \mathrm{~Hz})$ to the very top of their sustained range.

\section{MATERIALS AND METHODS}

\section{A. Subjects}

Twelve sopranos, from 18 to 29 years old, participated. Four were non-expert singers (NE1 to NE4), four others (AD1 to AD4) were advanced students and four (PR1 to PR4) were young professionals. These singers, who were selected for their ability to produce high pitches, had differing voice classifications (see Table I).

\section{B. Protocol and measurements}

In a first step, singers were asked to perform three or more glissandi on the vowel [a]. Each glissando was produced in a single breath, consisting in an ascending glide, up to the upper extent of their range, followed by a descending glide. These glissandi enabled us to detect the highest frequency that each singer was able to produce (see dashed lines in Table II), as well as frequencies where pitch jumps and changes in voice quality occurred.

Singers were then asked to sustain a single note for $4 \mathrm{~s}$, on an [a] vowel, with no change in pitch or loudness and with limited vibrato. Three measurements were made for each note. The protocol was limited to the vowel [a], as previous studies reported how $R 1$ of different intended vowels converge above E5 $(\sim 650 \mathrm{~Hz})$ toward the same high values. ${ }^{13}$ This was repeated on a diatonic scale from A4 $(\sim 440 \mathrm{~Hz})$ to the highest pitch they could sustain. For seven of the 12 singers, the maximum pitch that could be sustained for more than a second (represented by solid vertical lines in Table II) was lower than the highest pitch produced briefly at the top of glissandi (the dashed vertical lines in Table I). The difference was 1 or 2 tones (100 to $300 \mathrm{~Hz}$ ) for NE2, NE3, NE4 and PR2, and from 3 to 6 tones (400 to $1100 \mathrm{~Hz}$ ) for $\mathrm{AD} 1, \mathrm{AD} 2$ and AD3 (see Table II). Consequently, sustained productions were recorded above $\mathrm{C} 6\left(f_{0}>1050 \mathrm{~Hz}\right)$ for only eight singers, and above E6 for only five singers. Two 


\begin{tabular}{|c|c|c|c|c|}
\hline & & Age & Singing experience & $\begin{array}{l}\text { Self reported } \\
\text { classification }\end{array}$ \\
\hline \multirow[t]{4}{*}{ Nonexpert } & NE1 & 28 & Choir, singing lessons for 2 years & Light \\
\hline & NE2 & 28 & Previously trained, had not sung for 7 years & Coloratura \\
\hline & NE3 & 20 & Choir, singing lessons & Coloratura \\
\hline & NE4 & 18 & Choir & None \\
\hline \multirow[t]{4}{*}{ Advanced } & AD1 & 20 & Singing lessons for 8 years & Coloratura \\
\hline & AD2 & 19 & Singing lessons for 10 years & Lyrical \\
\hline & AD3 & 19 & Singing lessons for 6 years & Coloratura \\
\hline & AD4 & 20 & Singing lessons for 7 years & Dramatic \\
\hline \multirow[t]{4}{*}{ Professional } & PR1 & 26 & Singing lessons for 13 years, professional for 4 years & Coloratura \\
\hline & PR2 & 25 & Singing lessons for 7 years, professional for 1 year & Coloratura \\
\hline & PR3 & 29 & Singing lessons for 14 years, professional for 4 years & Coloratura \\
\hline & PR4 & 24 & Singing lessons for 9 years, professional for 2 years & Mezzo with coloratura top \\
\hline
\end{tabular}

singers demonstrated a pitch range (E5 to B5 for AD4 and B4 to B5 for NE1), over which they were able to produce very different voice qualities. These will be referred to as "full head" and "fluty resonant." Over this "overlap" range, these two singers were asked to produce 3 three occurrences of each note in both qualities.

Singers stood in front of a stand to which were attached a 1/4 in. pressure microphone (Brüel and Kjær 4944-A) and a small flexible tube, side by side. The tube was connected to a loudspeaker via an impedance matching horn and was used to excite the vocal tract with a synthesized broadband signal. The stand was adjusted for height so that the microphone and the flexible tube rested gently on the singer's lower lip throughout the experiment. A second identical pressure microphone was placed $30 \mathrm{~cm}$ away from the stand, and in front of the singer, as was a video camera (Panasonic DVC30). The audio signals from both microphones were pre-amplified (Brüel and Kjær Nexus 2690), then digitized at 16 bits and a rate of $44.1 \mathrm{kHz}$ using a Firewire audio interface (MOTU 828).

The singer's vocal tract was only excited by the broadband signal during the last three seconds of phonation. Thus, during the first second of phonation, the mean fundamental frequency $\left(f_{0}\right)$, the mean sound pressure level (SPL) and the average spectrum (calculated using 4096 points) were measured from the clean voice signal recorded $30 \mathrm{~cm}$ away from the singer's lips. The level of voice harmonics $\left(H_{i}\right.$, in $\left.\mathrm{dB}\right)$ was extracted from the average spectrum using MATLAB. The difference $r$ between the mean level of the two first harmonics and the mean level of the remaining harmonics below 10 $\mathrm{kHz}$ could then be calculated.

$$
r=\operatorname{mean}\left(H_{1}, H_{2}\right)-\operatorname{mean}\left(H_{3}, H_{i \leq 10 \mathrm{kHz}}\right) .
$$

The last $3 \mathrm{~s}$ of phonation were dedicated to the measurement of the first two vocal tract resonances, using an acoustic technique described in further detail by Epps et al. ${ }^{24}$ and Joliveau et al. $^{13}$ Briefly, the vocal tract was excited at the lips while the subject was singing, with a synthesized broadband signal consisting of a sum of sine waves over the range 200 to 3000 $\mathrm{Hz}$ with components spaced at $10.77 \mathrm{~Hz}\left(=44.1 \mathrm{kHz} / 2^{12}\right)$.
The tube with small internal diameter $(6 \mathrm{~mm})$ provided the source of acoustic flow. The microphone placed at lips, adjacent to the tube, recorded the vocal tract response to that excitation. The frequency of the first two vocal resonances was detected manually, by two of the authors, from the maxima of the measured pressure ratios

$$
\gamma=p_{\|} / p_{r},
$$

where $p_{\|}$is the pressure spectrum measured with the mouth open, and $p_{r}$ that measured at the lips with the mouth closed in an earlier calibration procedure. Because the source is a good approximation to a current source, this ratio is also effectively that of impedance of the tract at the mouth, in parallel with the radiation field, to that of the radiation field.

Following a previous labiometric system, ${ }^{25}$ images of the singer's lips were recorded from the front with the video camera, at a rate of 25 images/s. A grid marked in $10 \mathrm{~mm}^{2}$ was placed in the plane of the singer's mouth and recorded prior to the recording session in order to convert pixels into millimeters for the analysis of lip articulation. Because the lower lip maintained contact with the acoustic source and microphone, the lips were assumed to remain in the same plane throughout the experiment. Relative motion between mouth and camera could not be eliminated entirely, so that articulatory measurements obtained from that method have a precision around 5\%. Nevertheless, they yield information useful in interpreting acoustic measurements. Three articulatory parameters were extracted from the inner lip contour: Lip aperture (vertical opening), lip spreading (horizontal opening) and lip opening area are all defined in Fig. 2.

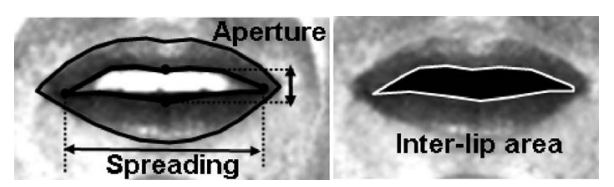

FIG. 2. A schematic showing the lip parameters extracted from frontal pictures of the singers. 


\section{RESULTS AND DISCUSSION}

Acoustical results are summarized in Table II. It indicates the limits of the pitch range over which each singer was able to sustain notes (in thick solid lines) and over which were measured lip articulation and vocal tract resonances. Notes below A4 $(\sim 440 \mathrm{~Hz})$ were not studied here. It also shows the additional range that some singers were able to produce, but on glissandi only (in dashed lines). The subranges over which tuning or proximity was observed between vocal tract resonances $(R 1, R 2)$ and voice harmonics $\left(f_{0}, 2 f_{0}\right)$ are shown by shading.

In Figs. 4-8, and later 11 and 12, the first panel shows a plot of the resonance frequencies as a function of fundamental frequency and pitch. The next panels show the parameters of lip geometry used to produce each note and the sound pressure levels produced.

\section{A. The region of $R 1: f_{0}$ tuning}

\section{1. $R 1: f_{0}$ tuning}

Figure 3 shows examples of the measured pressure ratio $\gamma$. In each case the fundamental of the sung pitch $f_{0}$ and its harmonics are visible as spikes superimposed over the measured broadband spectrum. Resonances in the vocal tract are

TABLE II. A summary of measurements: Thick solid lines delimit the pitch range over which each singer was able to sustain notes. Notes below A4 $(\sim 440 \mathrm{~Hz})$ were not studied here. Dashed lines show the limits of glissandi where this was greater. Shading shows the regions over which tuning or proximity was observed between vocal tract resonances $(R 1, R 2)$ and voice harmonics $\left(f_{0}, 2 f_{0}\right)$.
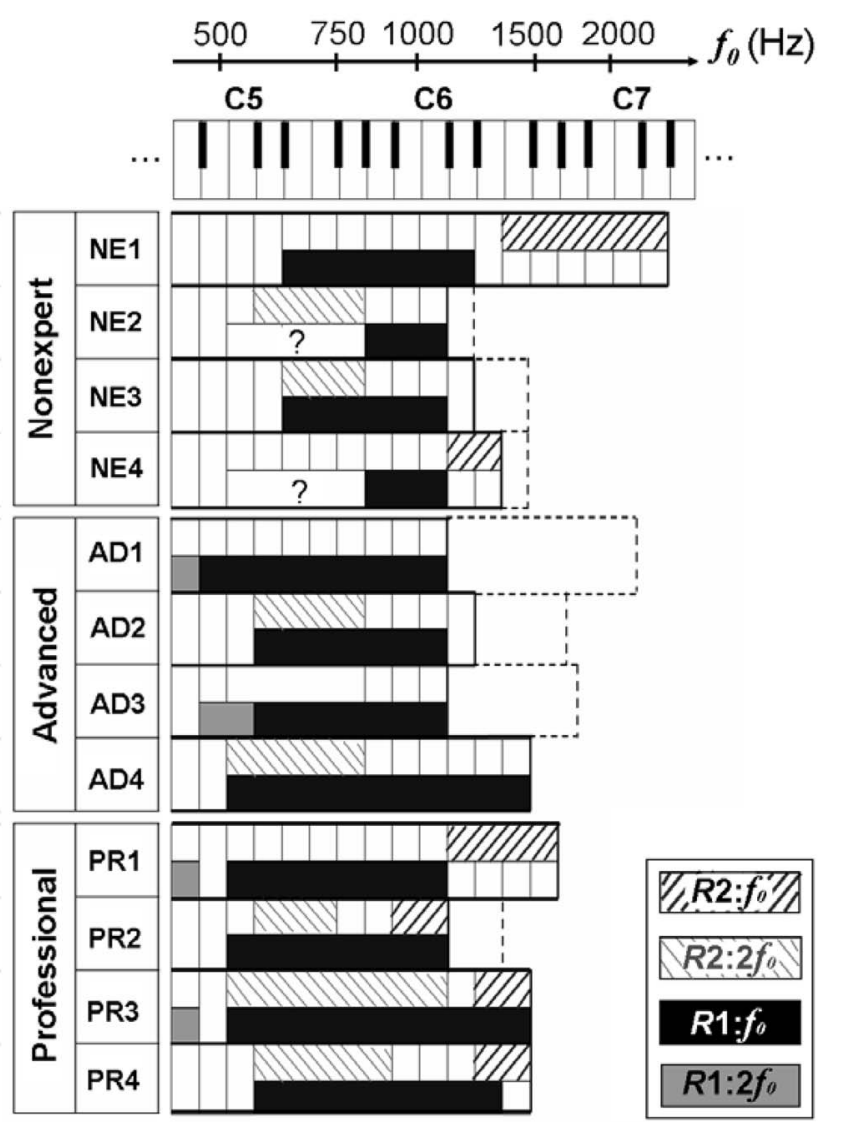

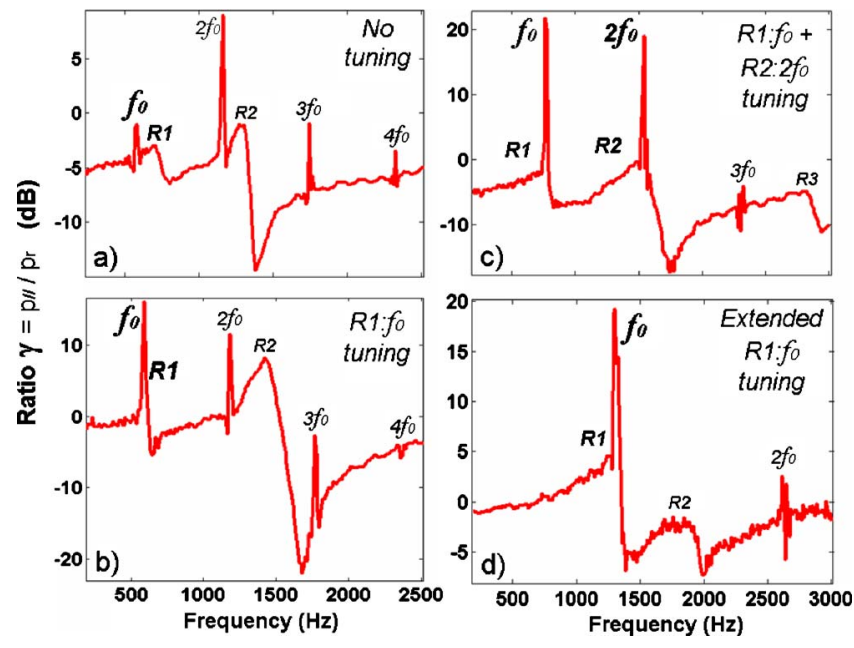

FIG. 3. (Color online) Examples of different resonance tuning strategies. Each figure shows the pressure ratio $\gamma$ measured as a function of frequency. In (a), no resonance adjustment is observed. In (b), the first vocal tract resonance $(R 1)$ is tuned to the first harmonic $\left(f_{0}\right)$. In $(\mathrm{c})$, the first and the second resonances $(R 1$ and $R 2)$ coincide with the first and second harmonics of the sung pitch $\left(f_{0}\right.$ and $2 f_{0}$ ). In (d), $R 1$ is increased to around $1300 \mathrm{~Hz}$ (F\#6), which is beyond the normal range of $R 1$ for speech.

associated with maxima in the broadband response. Figure 3(a) shows an example where $R 1, R 2, f_{0}$, and $2 f_{0}$ all occur at different frequencies, indicating that, in this case, there was no adjustment of resonances to harmonics. Figure 3(b) shows an example where $R 1$ coincides with $f_{0}$, indicating $R 1: f_{0}$ tuning.

All of the advanced students and professional singers studied were found to adjust $R 1$ to $f_{0}$ in a similar fashion when producing pitches over the range D5 to C6 $(\sim 600-1000 \mathrm{~Hz})$ - see Table II. The top panels of Figs. 5-7 illustrate how their $R 1$ values then follow the frequency of the first harmonic $\left(f_{0}\right)$.

Two of the non-expert singers, NE1 and NE3, also tuned $R 1$ to $f_{0}$ over the range $\mathrm{E} 5$ to $\mathrm{C} 6$, as shown in the top panels of Figs. 4 and 8. For the two remaining non-expert singers in this study (NE2 and NE4), $R 1$ was found close to $f_{0}$ for the small interval A5 to C6 (880 to $1000 \mathrm{~Hz})$. What happened for these singers below A5 is not clear. $R 1$ was hardly or not detectable from the pressure ratios $\gamma$ measured for these singers over C5 to A5 ( $\sim 450-900 \mathrm{~Hz})$, so that information about $R 1$ tuning is absent in that range.

a. The lower limit of $R 1: f_{0}$ tuning. The lower limit of $R 1: f_{0}$ tuning varied among singers. While a sustained region of $R 1: f_{0}$ tuning could be measured for two of the non-expert singers, they tended to start this tuning at higher pitch (E5 $\sim 650 \mathrm{~Hz})$ than did advanced students and professional singers (B4 to D5, i.e., $\sim 500-600 \mathrm{~Hz}$ ) - see Table II and top panels of Figs. $4-8$. This suggests that $R 1: f_{0}$ tuning is not necessarily an expert technique taught to classical singers. However, classically trained sopranos may learn to extend it to a lower range.

This difference in the lower limit for different singers was associated with the way in which $R 1$ varied with increasing $f_{0}$ around the onset of $R 1: f_{0}$ tuning.

For some singers (NE1, NE3, AD2, AD4, PR2, PR4), $R 1$ remained relatively constant for the low pitch range until its 


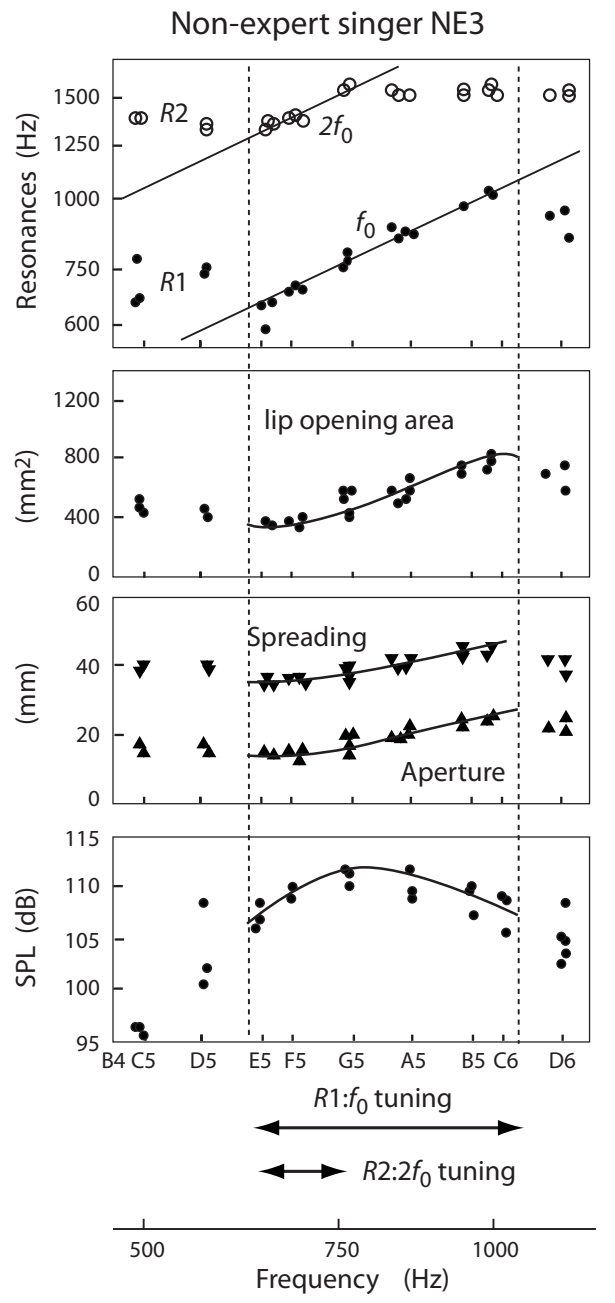

FIG. 4. The variation of vocal tract resonances $R 1$ and $R 2$ (top panel), lip articulation (second and third panels) and sound pressure level (SPL) (bottom panel) with increasing pitch for the non-expert singer NE3. The straight lines on the top panel indicate the relationships for $R 1: f_{0}$ tuning and $R 2: f_{0}$ tuning. The vertical dashed lines across the four panels indicate the range of $R 1: f_{0}$ tuning for this singer. The curves on the other panels only indicate the trends in the data over that $R 1: f_{0}$ tuning range.

value was reached by increasing $f_{0} . R 1$ then started following the first harmonic $\left(f_{0}\right)$ in a smooth way, exemplified in the top panel of Figs. 4, 7, and 8 .

For other singers (AD1, AD3, PR1, PR3), in the lowest range studied here (around $\mathrm{A} 4$ to $\mathrm{B} 4, \sim 450-500 \mathrm{~Hz}$ ), $R 1$ had a high value, which sometimes remained close to the second voice harmonic $\left(2 f_{0}\right)$ over a small pitch range. (Such a $R 1: 2 f_{0}$ adjustment was reported at similar pitch in Bulgarian female singing ${ }^{26}$ ). The top panels of Figs. 5 and 6 provide examples where $R 1$ decreased abruptly from $R 1 \cong 2 f_{0}$ on one pitch to $R 1 \cong f_{0}$ on the next pitch at the onset of $R 1: f_{0}$. Figure 9 gives examples of the pressure ratio $\gamma$ measured for singer PR3 (see also Fig. 6) and illustrates how $R 1$ can coincide with the second harmonic at A4 $(440 \mathrm{~Hz})$, but is then lowered so that it is nearer to the first harmonic at C5 (530 $\mathrm{Hz}$ ).

The singers who demonstrated a discontinuous behavior of $R 1$ around the start of $R 1: f_{0}$ tuning in the experiments on sustained notes are also those who had the least noticeable voice breaks or pitch instabilities in their glissandi over the

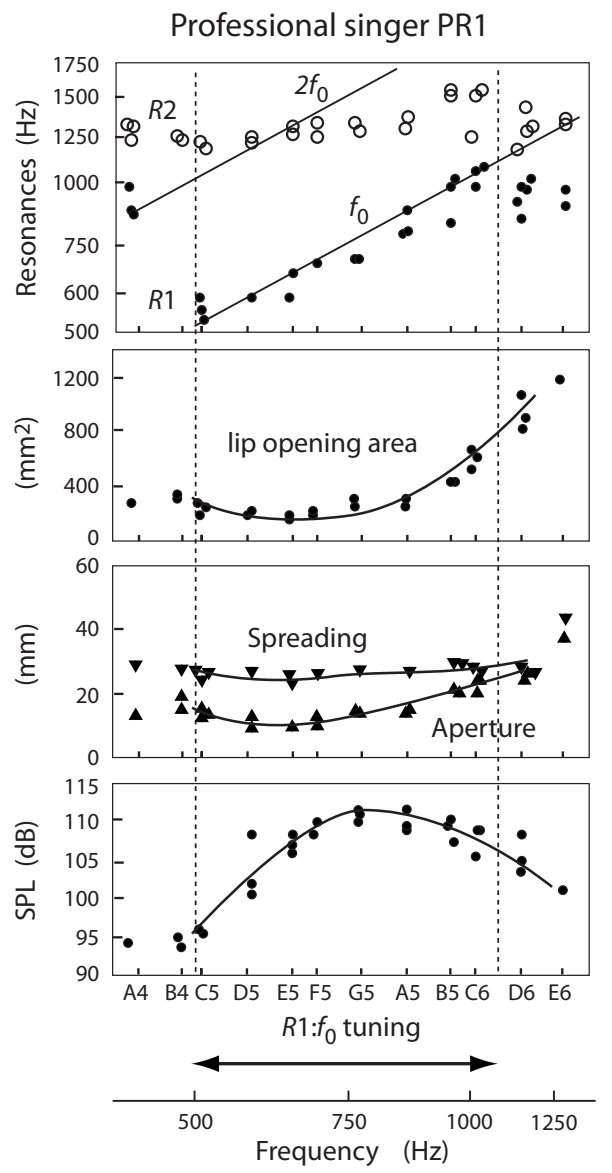

FIG. 5. The variation of vocal tract resonances $R 1$ and $R 2$, lip articulation and sound pressure level (SPL) with increasing pitch for the professional singer PR1 over the range from A4 to E6. See caption of Fig. 4 for further details.

passagio at D5 $(\sim 600 \mathrm{~Hz})$. Because resonances were not measured during the glissandi, the possibility that different resonance strategies are used in the two cases cannot be discounted. Nevertheless, it is tempting to suggest that the discontinuous behavior of $R 1$ is a strategy that helps avoid, over the D5 passagio, the potential instabilities that might result from sudden changes in the phase of the acoustic load in the range where the frequency difference $R 1-f_{0}$ changes sign.

$b$. The upper limit of $R 1: f_{0}$ tuning. The upper limit of $R 1: f_{0}$ tuning was less variable. Table II indicates that most of the singers (5 five out of 8 trained singers and all the non-experts) ceased tuning $R 1$ to $f_{0}$ around $\mathrm{C} 6$ to D6 ( $f$ $\sim 1100 \mathrm{~Hz}$ ) (see the top panels of Figs. 4, 5, and 8). For these singers, this corresponds to the highest or the second highest pitch they could sustain (solid vertical lines in Table II), although some of the singers could, in a glissando produce pitches from 2 tones to one octave higher (dashed lines in Table II).

Three singers (AD4, PR3 and PR4), however, were able to increase $R 1$ as high as $1300 \mathrm{~Hz}$. Figure 3(d) shows an example of the pressure ratio $\gamma$ where $R 1$ has been increased well above its range in normal speech. These three singers could thus extend their $R 1: f_{0}$ tuning up to E6 or F\#6 $(\sim 1300$ to $1500 \mathrm{~Hz}$ ), which was their upper limit for both sustained notes and glissandi (see Table II and the top panels of Figs. 6 and 7).

Garnier et al:: Articulatory strategies in high soprano range 3775 

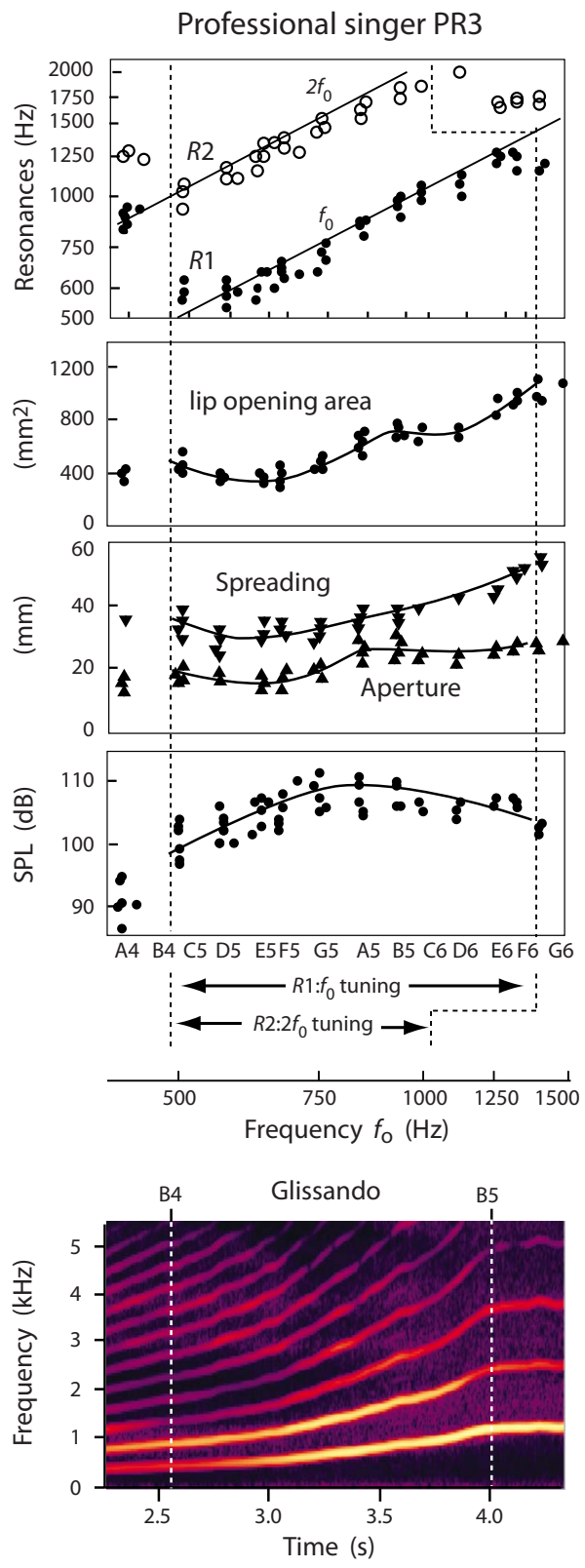

FIG. 6. (Color online) The variation of vocal tract resonances $R 1$ and $R 2$, lip articulation and sound pressure level (SPL) with increasing pitch for the professional singer PR3. The spectrogram of an ascending glissando produced by the same singer is shown underneath. See caption of Fig. 4 for further details.

\section{2. $R 2: 2 f_{0}$ tuning.}

For some singers (NE1, NE4, AD1 and PR1), the frequency of the second vocal tract resonance $(R 2)$ varied relatively little over the pitch range where $R 1: f_{0}$ tuning was evident (see the top panels of Figs. 5 and 8).

For all the other singers, $R 2$ increased over the range for which $R 1 \cong f_{0}$, in such a way that $R 2$ was close to the second voice harmonic $\left(2 f_{0}\right)$ over a range sometimes as small as two tones and sometimes as large as an octave (see top panel of Figs. 4, 6, and 7). Consequently, there was a pitch range over which both $R 1$ and $R 2$ lay close to $f_{0}$ and $2 f_{0}$, respectively (see Table II). Figure 3(c) gives an example of the pressure ratio $\gamma$ illustrating such a simultaneous $R 1: f_{0}$ and $R 2: 2 f_{0}$ tuning. The $R 2: 2 f_{0}$ tuning, observed here in some expert
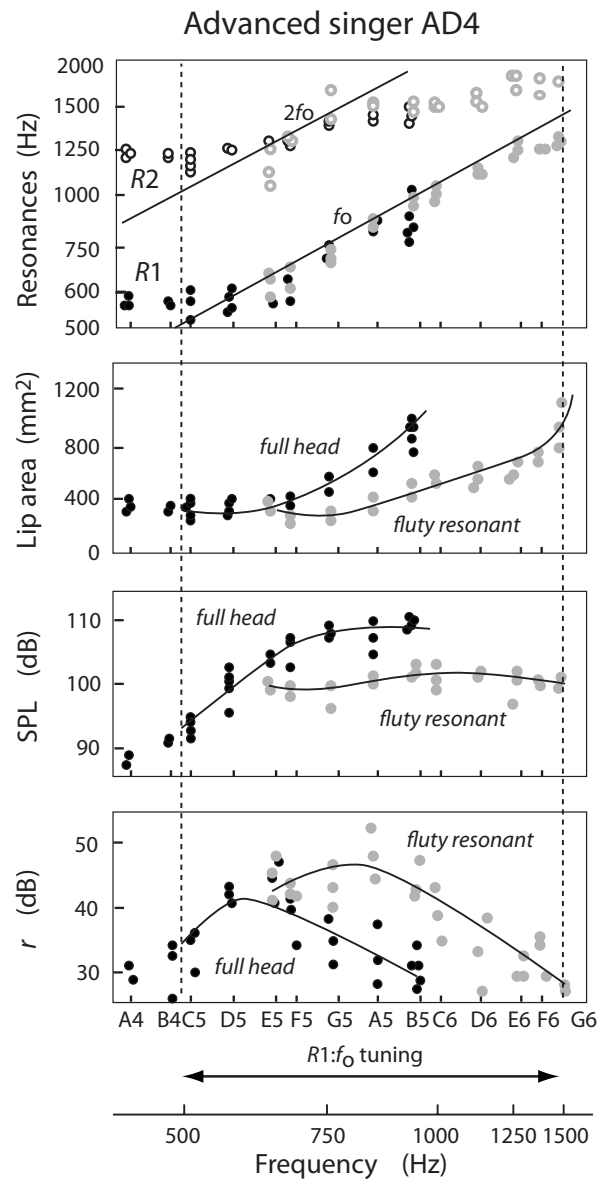

FIG. 7. Comparison of vocal tract resonances $R 1$ and $R 2$, lip articulation, sound pressure level (SPL,) and the difference $r$ in harmonic level [defined by Eq. (1)] between "full head" (black symbols) and "fluty resonant" (gray symbols) productions by advanced singer AD4. See caption to Fig. 4 for further details.

singers as well as non-expert ones, was not apparent in the average data reported in a previous study of resonance strategies in sopranos as it only occurred in two of their nine subjects. ${ }^{13}$

There were no direct measurements made of $R 1$ and $R 2$ during the glissandi produced by the same singers. However, it was observed that in glissandi, the levels $(H 1$ and $H 2)$ of the two first harmonics were enhanced with respect to those of the higher harmonics, over the frequency range where $R 1$ and $R 2$ were close to $f_{0}$ and $2 f_{0}$ on sustained pitches for the same singer (compare top panel and bottom spectrogram of Fig. 6). This is consistent with these singers adopting a similar resonance strategy in both glissandi and sustained pitches.

\section{Resonance tuning and voice quality}

For the two singers (NE1 and AD4) who could produce two different voice qualities or timbres over the range E5 to B5 $(\sim 650$ to $1000 \mathrm{~Hz})$, clear acoustical differences were found. The SPL was $10 \mathrm{~dB}$ weaker on average in the "fluty resonant" quality than in the "full head" one (see the third panel of Figs. 7 and 8). The "fluty resonant" quality also showed greater difference between the levels $(H 1$ and $H 2)$ of the two first harmonics and those of the higher harmonics, 

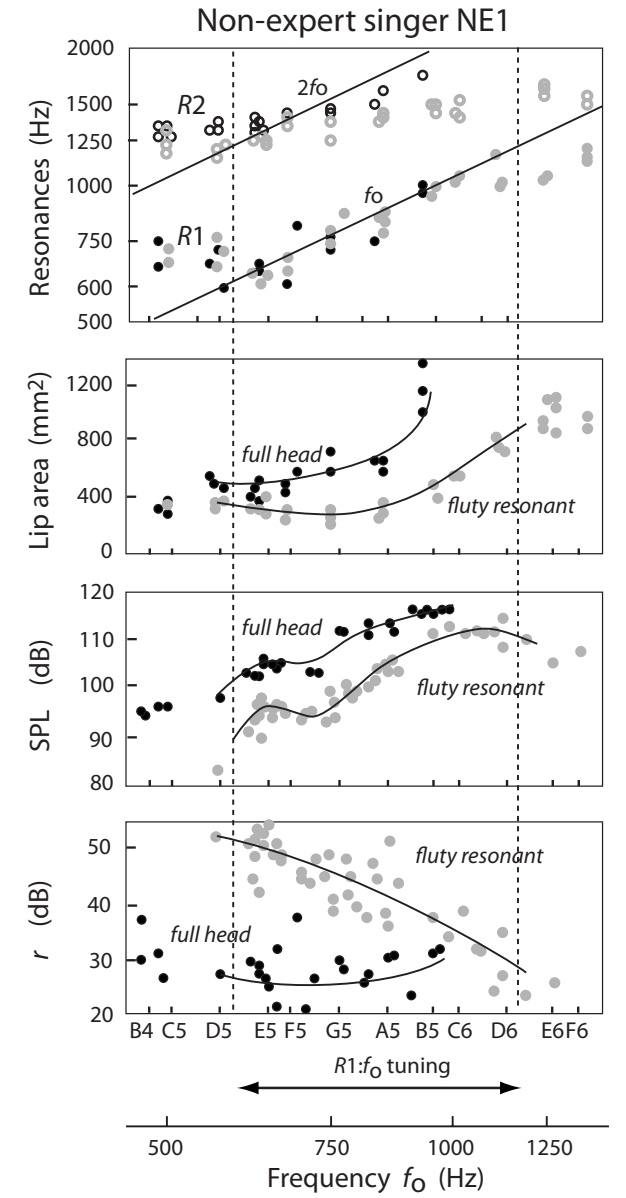

FIG. 8. Comparison of vocal tract resonances $R 1$ and $R 2$, lip articulation, sound pressure level (SPL) and the difference $r$ in harmonic level [defined by Eq. (1)] between "full head" (black symbols) and "fluty resonant" (gray symbols) productions by non-expert singer NE1. See caption of Fig. 4 for further details.

quantified by the parameter $r$ defined in Eq. (1) (see the bottom panels of Figs. 7 and 8). However, the behaviors of $R 1$ and $R 2$ over this range were similar for both qualities (see the first panel of Figs. 7 and 8), which supports the idea that differences in voice quality at high pitch are not primarily caused by the presence or the absence of tuning of the two first vocal tract resonances.

\section{The dependence of $R 1$ upon lip articulation}

a. Two different lip opening strategies. The singers we studied had differences in how they changed their lip opening with ascending pitch:
A4

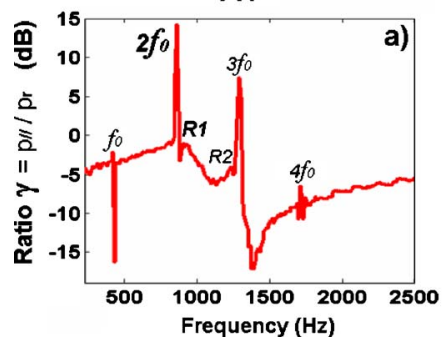

C5

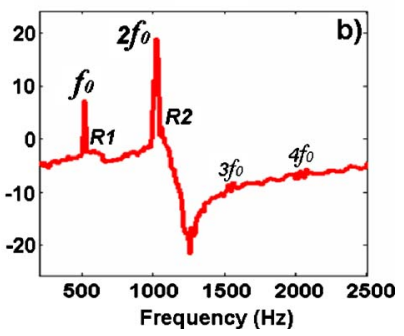

FIG. 9. (Color online) Comparison of the measured pressure ratio $\gamma$ for the production of the pitches A4 (a) and C5 (b) by the professional singer PR3. On A4 $(440 \mathrm{~Hz})$, the first resonance $R 1$ is adjusted to the second voice harmonic $\left(2 f_{0}\right)$. On C5 (about $500 \mathrm{~Hz}$ ), the frequencies of $R 1$ and $R 2$ are lowered considerably so they are close to $f_{0}$ and $2 f_{0}$, respectively.
Some singers (NE3, AD2, PR4) showed a nearly continuous increase of lip opening area over the whole pitch range in which they tuned $R 1$ to $f_{0}$ (see second panel of Fig. 4). Singer PR3 differed slightly from the others by showing a little plateau in the middle of that increase, between B5 and D6 ( 1000 to1200 Hz) (see second panel of Fig. 6).

Other singers showed a different lip-opening strategy: over the lower part of the $R 1: f_{0}$ tuning range, they did not vary the lip opening area-and sometimes even decreased it by $100-$ to $200 \mathrm{~mm}^{2}$ (approximately equivalent to the area of an 11 to $16 \mathrm{~mm}$ diameter circle). Thus, PR1 and PR2 reduced the area over the range from C5 to E5 $(\sim 500-650 \mathrm{~Hz})$ while AD3 reduced it over D5 to G5 $(\sim 600-800 \mathrm{~Hz})$. Only above this range did these singers use increasing lip opening area to achieve increases in $R 1$ with increasing pitch. The second panel of Fig. 5 provides an example. Evidently, these singers were using complementary articulators in addition to lips and jaw.

Non expert singers NE4 and NE2, who presented different behavior of $\gamma$ below A5 $\left(f_{0}<900 \mathrm{~Hz}\right)$, maintained relatively constant lip area and shape below A5 and C6 $(\sim 1000 \mathrm{~Hz})$ respectively.

Hence, the lip-opening strategy adopted over the range where $R 1$ increases did not depend simply on the singer's expertise.

However, the different articulatory strategies used to achieve $R 1: f_{0}$ tuning appeared to correlate with differences in intended voice quality. For the two singers who could produce both over a common range, the "full head" quality was produced with continuous increase of lip opening with increasing pitch, whereas the "fluty resonant" sound was produced with decreased lip opening (from E5 to A5 ( 650 to $900 \mathrm{~Hz}$ ) for singer NE1 and from C5 to G5 ( $\sim 500$ to 800 $\mathrm{Hz}$ ) by singer AD4, as shown in the second panel of Figs. 7 and 8).

b. Differences in lip opening. No significant differences between singers were observed in the extremes of lip opening over their $R 1: f_{0}$ range. Whatever the singer's expertise, lip opening area was typically around 300 to $400 \mathrm{~mm}^{2}$ (which would correspond to a circle of $\sim 20 \mathrm{~mm}$ diameter) at the beginning of $R 1: f_{0}$ tuning and around 800 to $1000 \mathrm{~mm}^{2}$ (corresponding to a circle of $36 \mathrm{~mm}$ diameter) at its end. Only NE1 and PR4 demonstrated a lip opening area greater than $1100 \mathrm{~mm}^{2}$ at the end of their $R 1: f_{0}$ tuning (see second panel of Fig. 8). In the case of PR4, this might partly explain how this singer was able to extend $R 1$ to a higher frequency than others and thus to keep on tuning it to $f_{0}$ up to F6. On the other hand, singers AD4 and PR3, who were also able to raise $R 1$ higher than $1000 \mathrm{~Hz}$, did not demonstrate a wider mouth than others. On the contrary, they demonstrated below D6 $\left(f_{0}<1200 \mathrm{~Hz}\right)$ a more closed mouth than others-with a lip opening area less than $800 \mathrm{~mm}^{2}$ (see the third panel of Fig. 6 as well as the "fluty resonant" quality on the second panel of Fig. 7). This smaller area did however leave them the possibility to continue increasing the area as the pitch rose above D6.

Greater differences among singers were observed in the shape of their inter-lip opening. 
To increase their lip opening area over the $R 1: f_{0}$ range, some singers only increased their vertical lip opening without changing lip spreading (NE4, AD3, AD4, PR1, PR2-See the third panel of Fig. 5 for an example). Others increased both vertical lip opening and spreading at the same time (NE1, NE3, AD2, PR4-See the third panel of Fig. 4 for an example). These different behaviors were not related to voice expertise, or to the two lip-opening strategies described above for $R 1: f_{0}$ tuning.

Interestingly, it was observed that the singer PR3 increased vertical lip opening up to B5, then maintained it constant and increased lip spreading only (see the third panel of Fig. 6). This transition pitch corresponds not only to the "plateau" observed in the increase of lip opening area see the second panel of Fig. 6) but also to the pitch from which this singer ceased the double resonance adjustment $\left(R 1: f_{0}\right.$ and $R 2: 2 f_{0}$ ) and extended the $R 1: f_{0}$ tuning alone (see the first panel of Fig. 6). However, the same lip strategy was not shown by other singers (NE2, NE3, AD2, AD3, AD4, PR2, PR4) who also demonstrated a proximity of $R 2$ to $2 f_{0}$ over the range of $R 1: f_{0}$ tuning (although over a narrower range than PR3).

c. Summary and discussion of the articulatory strategies for raising $R 1$. In summary, these results agree with the overall lowering of jaw and overall increasing of mouth opening with increasing pitch reported in trained sopranos $^{8-10,21}$ and with the similar increase of mouth opening in both expert and non-expert sopranos. ${ }^{22}$ However, some subjects, both expert and non-expert, did not increase lip opening-and sometimes even decreased it-over some parts of the $R 1: f_{0}$ range. Increasing $R 1$ with constant or decreasing lip opening area implies the involvement of other articulatory adjustments.

Furthermore, increasing the mouth opening to increase $R 1$ is expected to increase also $R 2$. However, the proportional effect of mouth opening on $R 2$ is smaller. ${ }^{27}$ So, where $R 2: 2 f_{0}$ and $R 1: f_{0}$ proximity extend over several notes, as for singer PR3 (see first panel of Fig. 6), this suggests again that (at least) one other articulation parameter, in addition to mouth opening, is being tuned.

If the vocal tract is considered as a simple duct that is almost closed at the glottis and open at the mouth, the frequency of the first resonance should be raised by increasing the cross section near the mouth. However, it should also be raised by the following four strategies:

(i) Reducing the overall length. Pabst and Sundberg ${ }^{28}$ observed that some trained sopranos raise their larynx in the high range. Johnson ${ }^{21}$ reported substantial rising $(40 \mathrm{~mm})$ in the production of high pitched "Swedish Kulning" technique, and correlations between larynx height and pitch. A shorter vocal tract anatomy may also be an advantage to raise $R 1$ to higher frequency.

(ii) Reducing the cross section of the pharyngeal cavity would seem to be contrary to the techniques of "yawning" and "covering" recommended in classical singing ${ }^{29-31}$ and contrary to recent MRI observations on trained sopranos. ${ }^{10}$ However, this would be coherent with the considerable pharyngeal constriction re-

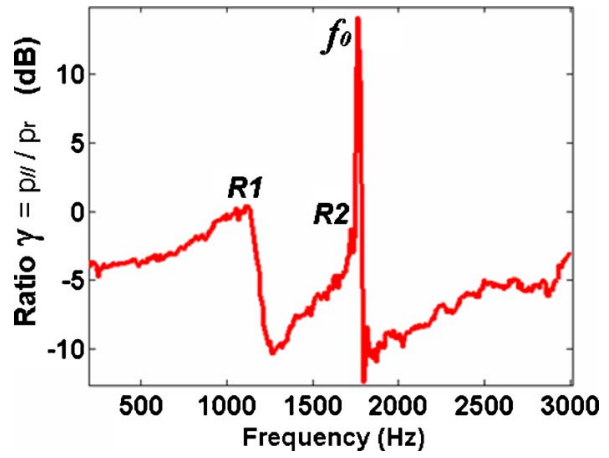

FIG. 10. (Color online) The measured pressure ratio $\gamma$ for the note A6 $(\sim 1750 \mathrm{~Hz})$ sung by the non-expert singer NE1. The second vocal tract resonance $R 2$ is adjusted to the first voice harmonic $f_{0}$.

ported in the whistle register of trained sopranos ${ }^{32}$ and in a professional kulner, ${ }^{21}$ which was enough to prevent these authors form conducting endoscopic examination of the glottis.

(iii) Increasing the average glottal opening or its open quotient would raise the frequency of $R 1 .^{33}$ Such an increase has been also reported in the transition from normal to whisper phonation. ${ }^{34}$

(iv) Appropriate changes in the topology of the duct. For example coupling of the nasal to the oral tract increases $R 1$ frequency $^{35,36}$ and is thought to help improve voice efficiency. ${ }^{37,38}$ Use of nasality in singing has already been reported in previous studies. ${ }^{39-41}$ However, Austin observed that the velo-pharyngeal opening tends to decrease with ascending pitch. ${ }^{42}$

\section{B. Above the region of $R 1: f_{0}$ tuning 1. $R 2: f_{0}$ tuning}

For half of the subjects (professionals as well as nonexperts), $f_{0}$ approached $R 2$ after the end of the $R 1: f_{0}$ tuning, i.e., around B5 $(\sim 1000 \mathrm{~Hz})$ for PR2 and around D6 to F6 $(\sim 1300 \mathrm{~Hz})$ for NE1, NE4, PR1, PR2 and PR4. In principle, this makes $R 2$ available to tune to $f_{0}$ in a range above that for which these singers use $R 1: f_{0}$ tuning (see Fig. 1). Did any of the singers use $R 2: f_{0}$ tuning?

Singer NE1 tuned $R 2$ near $f_{0}$ over the range F6 to D7 $(\sim 1400$ to $2300 \mathrm{~Hz})$ - see top panel of Fig. 11. An example of the observed pressure ratio $\gamma$ is given in Fig. 10 and illustrates how $R 2$ is adjusted to the first voice harmonic $\left(f_{0}\right)$. Singer PR 1 also showed $R 2$ close to or slightly above $f_{0}$ over the range D6 to G6 ( $\sim 1200$ to $1600 \mathrm{~Hz}$ ) (see top panel of Fig. 12). For PR2, PR4 and NE4, the notes above the $R 1: f_{0}$ tuning range also showed $R 2$ close to $f_{0}$. For PR3, $R 2$ exceeded $f_{0}$ by a few hundred $\mathrm{Hz}$ or less, i.e., $20 \%$ or less. For all these singers, the SPL was between 100 and $115 \mathrm{~dB}$ over that $R 2: f_{0}$ range (see bottom panel of Figs. 11 and 12). The end of the proximity between $R 2$ and $f_{0}$ corresponded to the limit of their ability to sustain notes (but not necessarily to produce higher pitches briefly in a glissando)—see Table II.

For singers PR1, PR2, PR4 and NE4, the transition between the $R 1: f_{0}$ tuning range and the range in which $R 2$ approximately equals $f_{0}$ was abrupt. From one diatonic note to the next, they switched from $R 1: f_{0}$ to $R 2: f_{0}$ (see Table II 


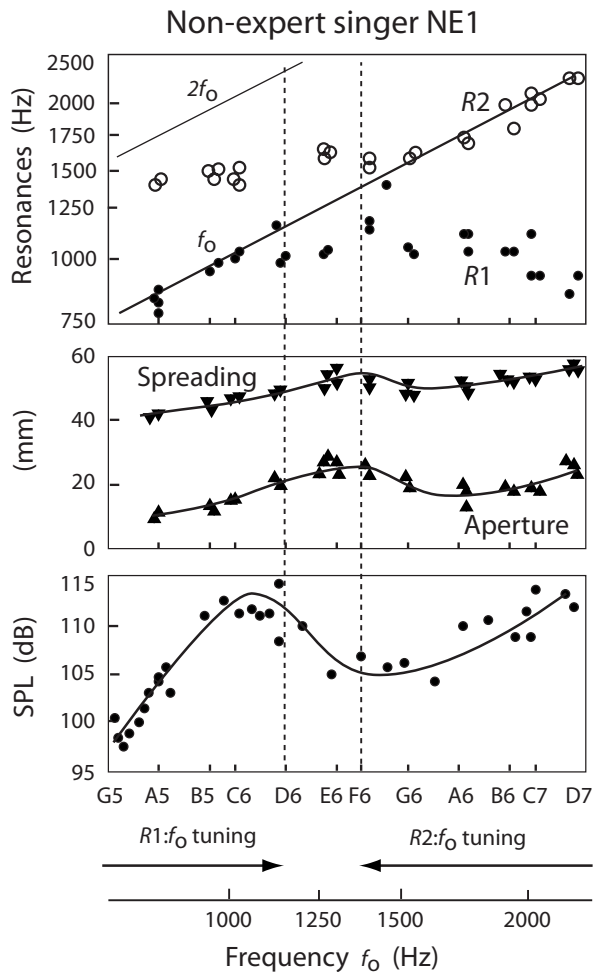

FIG. 11. The variation of vocal tract resonances $R 1$ and $R 2$, lip opening area and sound pressure level (SPL) with increasing pitch for the non-expert singer NE1 over the range G5 to D7. The vertical dashed lines across the three panels indicate the ranges of $R 1: f_{0}$ and $R 2: f_{0}$ tuning for this singer. See caption of Fig. 4 for further details.

and top panel of Fig. 12). For non-expert NE1, however, there were one or two diatonic notes that fell between these two tuning regimes (see top panel of Fig. 11). The sound level of these notes dropped by several $\mathrm{dB}$ once the $R 1: f_{0}$ range was exceeded, but increased again toward the end of her $R 2: f_{0}$ tuning (see bottom panel of Fig. 11).

Thus extending the $R 1: f_{0}$ tuning or alternatively tuning $R 2$ to $f_{0}$ appears to be a useful strategy to produce sustained notes in the extreme pitch range beyond D6. These strategies are not exclusive: one of the professional singers (PR4) demonstrated both the ability to extend the $R 1: f_{0}$ above D6 and to tune $R 2$ to $f_{0}$ from D6 $(\sim 1150 \mathrm{~Hz})$. Resonance adjustments may explain how loud and resonant sounds can still be produced over the top range, despite the weak vocal fold vibration reported in previous studies. ${ }^{11,20}$ However, they do not explain the difference in voice quality and in efficiency between trained coloratura sopranos and non-expert singers.

\section{Lip articulation during the $R 1: f_{0}$ to $R 2: f_{0}$ transition}

The transition from the $R 1: f_{0}$ to $R 2: f_{0}$ adjustment coincided with a change in lip opening. Below this transition, all singers increased lip aperture (vertical dimension) as $f_{0}$ increased. Above the transition, lip aperture was nearly constant for some singers (NE4, PR1, PR3, see the third panel of Fig. 12) and decreased over the transition for others (PR2, PR4, NE1, see the third panel of Fig. 11). Lip spreading (horizontal dimension) followed that same tendency, except for PR3 who kept on increasing lip spreading above C6

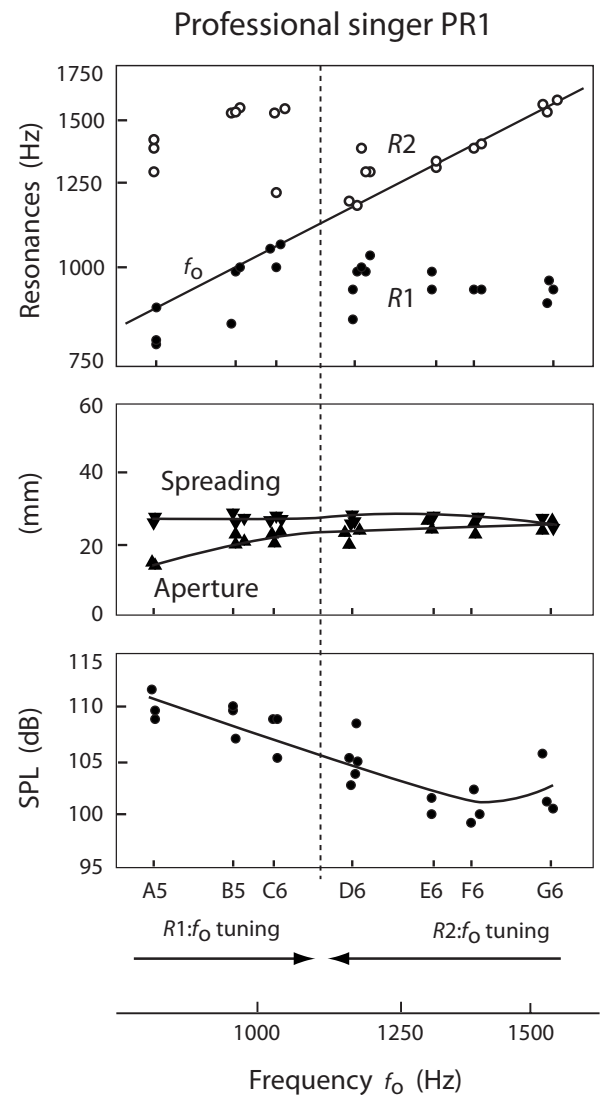

FIG. 12. The variation of vocal tract resonances $R 1$ and $R 2$, lip opening area and sound pressure level (SPL) with increasing pitch for the professional singer PR1 over the range A5 to G6. The vertical dashed lines across the three panels indicate the ranges of $R 1: f_{0}$ and $R 2: f_{0}$ tuning for this singer. See caption of Fig. 4 for further details.

$(\sim 1050 \mathrm{~Hz})$ (see the third panel of Fig. 6). This may explain why she showed proximity between $R 2$ and $f_{0}$, but no precise adjustment. Several tones above the transition, NE1 started increasing lip aperture and spreading again, from A6 to $\mathrm{D} 7$ ( $\sim 1800$ to $2300 \mathrm{~Hz}$ ) (see the second panel Fig. 11).

Finally, this report has concentrated on vocal tract adjustments in the high soprano range. The glottal behavior and its relationship with vocal-tract adjustments remains an open question.

\section{CONCLUSIONS}

$R 1: f_{0}$ tuning is observed not only in trained singers, but also in non-experts. However, the expert singers began this tuning at lower pitches. Some singers can combine $R 2: 2 f_{0}$ adjustment with $R 1: f_{0}$ tuning.

Two different lip-opening strategies are used to achieve $R 1: f_{0}$ tuning. Some singers continuously increase lip opening with increasing pitch whereas other increase it only over the highest part of the $R 1: f_{0}$ range. This strongly suggests the involvement of other articulators in $R 1: f_{0}$ tuning.

Expert and non-expert singers who were able to sustain pitches above C6 to D6 $\left(f_{0}>1100 \mathrm{~Hz}\right)$ either extended the $R 1: f_{0}$ tuning into this range, or made a transition to $R 2: f_{0}$ tuning.

Garnier et al:: Articulatory strategies in high soprano range 3779 


\section{ACKNOWLEDGMENT}

We thank our volunteer subjects and the Australian Research Council for support.

${ }^{1}$ M. Garcia, Mémoire sur la Voix Humaine Présenté à l'académie des Sciences en 1840 (Memoire on the Human Voice presented at the Sciences Academy in 1840), 2nd ed. (Imprimerie E. Duverger, Paris, 1847).

${ }^{2}$ H. Hollien, "On vocal registers," J. Phonetics 2, 125-143 (1974).

${ }^{3} \mathrm{~N}$. Henrich, "Mirroring the voice from Garcia to the present day: Some insights into singing voice registers," Logoped. Phoniatr. Vocol. 31, 3-14 (2006).

${ }^{4}$ D. G. Miller, Registers in Singing: Empirical and Systematic Studies in the Theory of the Singing Voice (University of Groningen, Groningen, The Netherlands, 2000).

${ }^{5}$ B. Roubeau, N. Henrich, and M. Castellengo, "Laryngeal vibratory mechanisms: The notion of vocal register revisited," J. Voice 23, 425-438 (2009).

${ }^{6}$ J. Švec, H. K. Schutte, and D. G. Miller, "On pitch jumps between chest and falsetto registers in voice: Data from living and excised human larynges," J. Acoust. Soc. Am. 106, 1523-1531 (1999).

${ }^{7}$ B. Roubeau, M. Castellengo, P. Bodin, and M. Ragot, "Phonétogramme par registre laryngé (Laryngeal registers as shown in the voice range profile)," Folia Phoniatr Logop 56, 321-333 (2004).

${ }^{8}$ J. Sundberg and J. Skoog, "Dependence of jaw opening on pitch and vowels singers," J. Voice 11, 301-306 (1997).

${ }^{9}$ J. Sundberg, "Articulatory configuration and pitch in a classically trained soprano singer," J. Voice 23, 546-551 (2009).

${ }^{10}$ M. Echternach, J. Sundberg, S. Arndt, M. Markl, M. Schumacher, and B. Richter, "Vocal tract in female registers-A dynamic real-time MRI study," J. Voice 24, 133-139 (2010).

${ }^{11}$ D. G. Miller and H. K. Schutte, "Physical definition of the "flageolet register"," J. Voice 7, 206-212 (1993).

${ }^{12} \mathrm{~J}$. Sundberg, "Formant technique in a professional female singer," Acustica 32, 89-96 (1975).

${ }^{13}$ E. Joliveau, J. Smith, and J. Wolfe, "Vocal tract resonances in singing: The soprano voice," J. Acoust. Soc. Am. 116, 2434-2439 (2004).

${ }^{14}$ E. Joliveau, J. Smith, and J. Wolfe, "Tuning of vocal tract resonance by sopranos," Nature (London) 427, 116 (2004).

${ }^{15}$ B. E. Lindblom and J. E. Sundberg, "Acoustical consequences of lip, tongue, jaw, and larynx movement," J. Acoust. Soc. Am. 50, 1166-1179 (1971).

${ }^{16}$ J. Sundberg, The Science of the Singing Voice (Northern Illinois University Press, DeKalb, IL, 1987).

${ }^{17}$ I. R. Titze, Principles of Voice Production (Prentice-Hall, Englewood Cliffs, NJ, 1994).

${ }^{18}$ I. R. Titze, "The Physics of small-amplitude oscillations of the vocal folds," J. Acoust. Soc. Am. 83, 1536-1552 (1988).

${ }^{19}$ I. R. Titze, "Nonlinear source-Filter coupling in phonation: Theory," J. Acoust. Soc. Am. 123, 2733-2749 (2008)

${ }^{20} \mathrm{M}$. Rothenberg, "Acoustic reinforcement of vocal fold vibratory behavior in singing," in Voice Physiology: Voice Production, Mechanisms and Functions. Raven, New York, 1988), pp. 379-390.

${ }^{21}$ A. Johnson, "Voice physiology and ethnomusicology: Physiological and acoustical studies of the Swedish herding song," Yearb. Tradit. Music 16, 42-66 (1984)
${ }^{22}$ S. F. Austin, "Jaw opening in novice and experienced classically trained singers," J. Voice 21, 72-79 (2007).

${ }^{23}$ L. Thurman, G. Welch, A. Theimer, and C. Klitzke, "Addressing vocal register discrepancies: An alternative, science-based theory of register phenomena," in Proceedings of the 2nd International Conference of The Physiology and Acoustics of Singing, Denver, CO (2004).

${ }^{24}$ J. Epps, J. R. Smith, and J. Wolfe, "A novel instrument to measure acoustic resonances of the vocal tract during speech," Meas. Sci. Technol. 8, 1112-1121 (1997).

${ }^{25}$ M. T. Lallouache, "Un poste 'visage-parole'. acquisition et traitement de contours labiaux (a 'face-speech' system of acquisition and processing of lip contours)," in Proceedings of the 18th Journées d'Etudes sur la Parole, Montréal, Canada (1990), pp. 282-286.

${ }^{26}$ N. Henrich, M. Kiek, J. Smith, and J. Wolfe, "Resonance strategies used in Bulgarian women's singing style: A pilot study," Logoped. Phoniatr. Vocol. 32, 171-177 (2007).

${ }^{27}$ B. S. Rosner and J. B. Pickering, Vowel Perception and Production (Oxford University Press, New York, 1975).

${ }^{28}$ F. Pabst and J. Sundberg, "Tracking multi-channel electroglottograph measurement of larynx height in singers," STL-QPSR 33, 67-78 (1992).

${ }^{29}$ S. Hertegård, J. Gauffin, and J. Sundberg, "Open and covered singing as studied by means of fiberoptics, inverse filtering, and spectral analysis," J. Voice 4, 220-230 (1990).

${ }^{30}$ H. F. Mitchell and D. T. Kenny, "The impact of 'open throat' technique on vibrato rate, extent and onset in classical singing," Logoped. Phoniatr. Vocol. 29, 171-182 (2004).

${ }^{31}$ I. R. Titze, C. C. Bergan, E. J. Hunter, and B. Story, "Source and filter adjustments affecting the perception of the vocal qualities twang and yawn," Logoped. Phoniatr. Vocol. 28, 147-155 (2003).

${ }^{32}$ J. Švec, J. Sundberg, and S. Hertegård, "Three registers in an untrained female singer analyzed by videokymography, strobolaryngoscopy and sound spectrography," J. Acoust. Soc. Am. 123, 347-353 (2008).

${ }^{33}$ A. Barney, A. De Stefano, and N. Henrich, "The effects of glottal opening on the acoustic response of the vocal tract," Acta. Acust. Acust. 93, 10461056 (2007)

${ }^{34}$ Y. Swerdlin, J. Smith, and J. Wolfe, "The effect of whisper and creak vocal mechanisms on vocal tract resonances," J. Acoust. Soc. Am. 127, 2590-2598 (2010).

${ }^{35} \mathrm{G}$. Feng and E. Castelli, "Some acoustic features of nasal and nasalized vowels: A target for vowel nasalization," J. Acoust. Soc. Am. 99, 36943706 (1996)

${ }^{36}$ M. Y. Chen, "Acoustic correlates of English and French nasalized vowels," J. Acoust. Soc. Am. 102, 2360-2370 (1997).

${ }^{37}$ I. R. Titze, "Acoustic interpretation of resonant voice," J. Voice 15, 519$528(2001)$

${ }^{38}$ A. M. Laukkanen, P. Lindholm, and E. Vilkman, "On the effects of various vocal training methods on glottal resistance and efficiency. A preliminary report," Folia Phoniatr Logop 47, 324-330 (1995).

${ }^{39}$ N. Scotto di Carlo and D. Autesserre, "Movements of the velum in singing," J. Res. Sing. 11, 3-13 (1987).

${ }^{40}$ P. Birch, B. Gümoes, H. Stavad, S. Prytz, E. Björkner, and J. Sundberg, "Velum behavior in professional classic operatic singing," J. Voice 16, 61-71 (2002).

${ }^{41}$ J. Sundberg, P. Birch, B. Gümoes, H. Stavad, S. Prytz, and A. Karle, "Experimental findings on the nasal tract resonator in singing," J. Voice 21, 127-137 (2007).

${ }^{42} \mathrm{~S}$. F. Austin, "Movement of the velum during speech and singing in classically trained singers," J. Voice 11, 212-221 (1997). 